\title{
Designs, groups and lattices
}

\author{
par Christine BACHOC
}

\begin{abstract}
RÉSUMÉ. La notion de designs dans les espaces Grassmanniens a été introduite par l'auteur et R. Coulangeon, G. Nebe dans [3]. Après avoir rappelé les premières propriétés de ces objets et les relations avec la théorie des réseaux, nous montrons que la famille des réseaux de Barnes-Wall contient des 6-designs grassmanniens. Nous discutons également des relations entre cette notion de designs et les designs associés à l'espace symétrique formé des espaces totalement isotropes d'un espace quadratique binaire, qui sont mises en évidence par une certaine construction utilisant le groupe de Clifford.
\end{abstract}

Abstract. The notion of designs in Grassmannian spaces was introduced by the author and R. Coulangeon, G. Nebe, in [3]. After having recalled some basic properties of these objects and the connections with the theory of lattices, we prove that the sequence of Barnes-Wall lattices hold 6-Grassmannian designs. We also discuss the connections between the notion of Grassmannian design and the notion of design associated with the symmetric space of the totally isotropic subspaces in a binary quadratic space, which is revealed in a certain construction involving the Clifford group.

\section{Introduction}

Roughly speaking, a design is a finite subset of a space $X$ which "approximates well" $X$. In the case of finite spaces $X$, such objects arose from different contexts like statistics, finite geometries, graphs, and are well understood in the framework of association schemes ([6], [15]). Later the notion of designs was extended to the two-point homogeneous real manifolds ([14]). Of special interest are the so-called spherical designs, defined on the unit sphere of the Euclidean space. Due mainly to the work of Boris Venkov ([29]), we know that nice spherical designs arise from certain families of lattices, and that the lattices which contain spherical designs are locally dense. Moreover, this combinatorial property gives a hint to classify these lattices, which was recently fulfilled in many cases ([5]).

In a common work with $\mathrm{R}$. Coulangeon and $\mathrm{G}$. Nebe, we have generalized these notions to the real Grassmannian spaces $\mathcal{G}_{m, n}$. This was the 
subject of my talk at the XXIIIèmes Journées Arithmétiques (2003), in Graz. I have chosen not to reproduce this talk here, but rather to present some complementary results on one aspect of this subject, which was not emphasized in Graz, namely the links with group representation. In particular, we will not discuss at all here the connections with Siegel modular forms.

Sections 2 to 4 essentially review on results from [3]. The zonal polynomials associated with the action of the orthogonal group on $\mathcal{G}_{m, n}$, which are generalized Jacobi polynomials in $m$ variables, play a crucial role. They are presented in $\S 2$. The existence of Grassmannian designs in a lattice is connected to its Rankin functions $\gamma_{m, n}$, this is recalled in $\S 3$. In $\S 4$, we recall how certain properties of the representations of a finite subgroup of $O\left(\mathbb{R}^{n}\right)$ ensures that its orbits on $\mathcal{G}_{m, n}$ are designs. This is successfully applied to the automorphism group of many lattices.

In $\S 5$, we introduce the Clifford groups $\mathcal{C}_{k}<O\left(\mathbb{R}^{2^{k}}\right)$, and their subgroups $\mathcal{G}_{k}$, of index 2 , which are the automorphism groups of the Barnes-Wall lattices. These groups have recently attracted attention in combinatorics because of their appearance in several apparently disconnected situations (finite geometries, quantum codes, lattices, Kerdock codes..). In [20], a very nice combinatorial proof that their polynomial invariants are spanned by the generalized weight enumerators of binary codes is given. We partly extend this result to the subgroup $\mathcal{G}_{k}$. As a consequence, we obtain that the Barnes-Wall lattices support Grassmannian 6-designs, and that they are local maxima for all the Rankin constants.

The last section, $\S 6$, discusses some other constructions of Grassmannian designs associated with the Clifford groups. We encounter another notion of design, this time associated with the space of totally isotropic subspaces of fixed dimension in a binary quadratic space. This space is homogeneous and symmetric for the action of the corresponding binary orthogonal group. Unsurprisingly, the Clifford group connects these two notions of designs, leading to interesting new examples of Grassmannian designs.

\section{Grassmannian designs}

2.1. Definitions. The notion of Grassmannian designs was introduced in [3]. Let $m \leq n / 2$, and let $\mathcal{G}_{m, n}$ be the real Grassmannian space, together with the transitive action of the real orthogonal group $\mathrm{O}\left(\mathbb{R}^{n}\right)$. The starting point is the decomposition of the space of complex-valued squared module integrable functions $L^{2}\left(\mathcal{G}_{m, n}\right)$ under the action of $\mathrm{O}\left(\mathbb{R}^{n}\right)$. One has:

$$
L^{2}\left(\mathcal{G}_{m, n}\right)=\oplus_{\mu} \mathrm{H}_{m, n}^{2 \mu}
$$


where the sum is over the partitions $\mu=\mu_{1} \geq \cdots \geq \mu_{m} \geq 0$, and the spaces $\mathrm{H}_{m, n}^{2 \mu}$ are isomorphic to the irreducible representation of $\mathrm{O}\left(\mathbb{R}^{n}\right)$ canonically associated with $2 \mu$, and denoted $V_{n}^{2 \mu}$ (see [16]). Here $2 \mu=2 \mu_{1} \geq \cdots \geq$ $2 \mu_{m} \geq 0$ is a partition with even parts. The degree of the partition $\mu$ is by definition $\operatorname{deg}(\mu):=\sum_{i} \mu_{i}$ and its length $l(\mu)$ is the number of its non-zero parts.

As an example, when $l(\mu)=1$, the representation $V_{n}^{\mu}$ is isomorphic to the space of polynomials in $n$ variables, homogeneous of degree $\mu_{1}$, and harmonic, i.e. annihilated by the standard Laplace operator. When $l(\mu)>1$, the representations $V_{n}^{\mu}$ have more complicated but still explicit realizations as spaces of polynomials in matrix arguments.

Definition ([3]). A finite subset $\mathcal{D}$ of $\mathcal{G}_{m, n}$ is called a $2 t$-design if, for all $f \in \mathrm{H}_{m, n}^{2 \mu}$ and all $\mu$ with $0 \leq \operatorname{deg}(\mu) \leq t$,

$$
\int_{\mathcal{G}_{m, n}} f(p) d p=\frac{1}{|\mathcal{D}|} \sum_{x \in \mathcal{D}} f(x) .
$$

The decomposition (2.1) immediately shows that this definition is equivalent to the condition:

$$
\text { for all } f \in \mathrm{H}_{m, n}^{2 \mu} \text { and all } \mu \text { with } 1 \leq \operatorname{deg}(\mu) \leq t, \sum_{x \in \mathcal{D}} f(x)=0 \text {. }
$$

There is a nice characterization of the designs in terms of the zonal functions of $\mathcal{G}_{m, n}$, which is much more satisfactory from the algorithmic point of view. We briefly recall it here.

It is a classical fact that the orbits under the action of $\mathrm{O}\left(\mathbb{R}^{n}\right)$ of the pairs $\left(p, p^{\prime}\right)$ of elements of $\mathcal{G}_{m, n}$ are characterized by their so-called principal angles $\left(\theta_{1}, \ldots, \theta_{m}\right) \in[0, \pi / 2]^{m}$. We set $y_{i}:=\cos ^{2}\left(\theta_{i}\right)$. The polynomial functions on $\mathcal{G}_{m, n} \times \mathcal{G}_{m, n}$ which are invariant under the simultaneous action of $\mathrm{O}\left(\mathbb{R}^{n}\right)$ are polynomials in the variables $\left(y_{1}, \ldots, y_{m}\right)$, and their space is isomorphic to the algebra $\mathbb{C}\left[y_{1}, \ldots, y_{m}\right]^{S_{m}}$ of symmetric polynomials in $m$ variables. Moreover, there is a unique sequence of orthogonal polynomials $P_{\mu}\left(y_{1}, \ldots, y_{m}\right)$ indexed by the partitions of length $m$, such that $\mathbb{C}\left[y_{1}, \ldots, y_{m}\right]^{S_{m}}=\oplus_{\mu} \mathbb{C} P_{\mu}, P_{\mu}(1, \ldots, 1)=1$, and the function : $p \in \mathcal{G}_{m, n} \rightarrow P_{\mu}\left(y_{1}\left(p, p^{\prime}\right), \ldots, y_{m}\left(p, p^{\prime}\right)\right)$ defines, for all $p^{\prime} \in \mathcal{G}_{m, n}$, an element of $\mathrm{H}_{m, n}^{2 \mu}$. These polynomials have degree $\operatorname{deg}(\mu)$. They are explicitly calculated in [17], where it is shown that they belong to the family of Jacobi polynomials.

More precisely, James and Constantine show that the canonical measure on $\mathcal{G}_{m, n}$, induces on $\mathbb{C}\left[y_{1}, \ldots, y_{m}\right]^{S_{m}}$ the following measure: 


$$
d \mu\left(y_{1}, \ldots, y_{m}\right)=\lambda \prod_{1 \leq i<j \leq m}\left|y_{i}-y_{j}\right| \prod_{1 \leq i \leq m} y_{i}^{-1 / 2}\left(1-y_{i}\right)^{n / 2-m-1 / 2} d y_{i}
$$

(where $\lambda$ is chosen so that $\int_{[0,1]^{m}} d \mu\left(y_{1}, \ldots, y_{m}\right)=1$ ). This measure defines an hermitian product on $\mathbb{C}\left[y_{1} \ldots, y_{m}\right]^{S_{m}}$, namely

$$
[f, g]=\int_{[0,1]^{m}} f(y) \overline{g(y)} d \mu(y) .
$$

Since the irreducible subspaces $\mathrm{H}_{m, n}^{2 \mu}$ are pairwise orthogonal, the corresponding polynomials $P_{\mu}$ must be orthogonal for this hermitian product. Together with some knowledge on the monomials of $\operatorname{degree} \operatorname{deg}(\mu)$ that occur in $P_{\mu}$, it is enough to uniquely determine them. However, the most efficient way to calculate them is to exploit the fact that they are eigenvectors for the operator on $\mathbb{C}\left[y_{1}, \ldots, y_{m}\right]^{S_{m}}$ induced by the Laplace-Beltrami operator (see [17], [3] for more details).

The first ones are equal to:

$$
\begin{gathered}
P_{0}=1 \\
P_{(1)}=\frac{1}{\beta_{1}}\left(\sum y_{i}-\frac{m^{2}}{n}\right), \quad \beta_{1}=m\left(1-\frac{m}{n}\right) \\
P_{(11)}=\frac{1}{\beta_{11}}\left(\sum y_{i} y_{j}-\frac{(m-1)^{2}}{n-2} \sum y_{i}+\frac{m^{2}(m-1)^{2}}{2(n-1)(n-2)}\right), \\
\beta_{11}=\frac{m(m-1)}{2}\left(1-2 \frac{m-1}{n-2}+\frac{m(m-1)}{(n-1)(n-2)}\right) \\
P_{(2)}=\frac{1}{\beta_{2}}\left(\sum y_{i}^{2}+\frac{2}{3} \sum y_{i} y_{j}-\frac{2(m+2)^{2}}{3(n+4)} \sum y_{i}+\frac{m^{2}(m+2)^{2}}{3(n+2)(n+4)}\right), \\
\beta_{2}=\frac{m(m+2)}{3}\left(1-2 \frac{m+2}{n+4}+\frac{m(m+2)}{(n+2)(n+4)}\right)
\end{gathered}
$$

where $\sum y_{i}=\sum_{1 \leq i \leq m} y_{i}, \sum y_{i}^{2}=\sum_{1 \leq i \leq m} y_{i}^{2}, \sum y_{i} y_{j}=\sum_{1 \leq i<j \leq m} y_{i} y_{j}$.

Theorem 2.1 ([3]). Let $\mathcal{D} \subset \mathcal{G}_{m, n}$ be a finite set. Then,

(1) for all $\mu, \sum_{p, p^{\prime} \in \mathcal{D}} P_{\mu}\left(y_{1}\left(p, p^{\prime}\right), \ldots, y_{m}\left(p, p^{\prime}\right)\right) \geq 0$.

(2) The set $\mathcal{D} \subset \mathcal{G}_{m, n}$ is a $2 t$-design if and only if for all $\mu$, $1 \leq \operatorname{deg}(\mu) \leq t, \sum_{p, p^{\prime} \in \mathcal{D}} P_{\mu}\left(y_{1}\left(p, p^{\prime}\right), \ldots, y_{m}\left(p, p^{\prime}\right)\right)=0$.

Remark. The first property is basic to the so-called linear programming method to derive bounds for codes and designs (see [2]). 
2.2. Some subsets of $\mathcal{G}_{m, n}$ associated with a lattice. Let $L \subset \mathbb{R}^{n}$ be a lattice. We define certain natural finite subsets of $\mathcal{G}_{m, n}$ associated with $L$, in the following way. Let $S_{m}(\mathbb{R}), S_{m}^{>0}(\mathbb{R}), S_{m}^{\geq 0}(\mathbb{R})$ be the spaces of $m \times m$ real symmetric, respectively real positive definite, and real positive semi-definite matrices.

Definition. Let $S \in \mathrm{S}_{m}^{>0}(\mathbb{R})$. Let $L_{S}$ be the set of $p \in \mathcal{G}_{m, n}$ such that $p \cap L$ is a lattice, having a basis $\left(v_{1}, \ldots, v_{m}\right)$ with $v_{i} \cdot v_{j}=S_{i, j}$ for all $1 \leq i, j \leq m$.

Clearly, the sets $L_{S}$ are finite sets. In the case $m=1$, the sets $L_{S}$ are the sets of lines supporting the lattice vectors of fixed norm.

Definition. Let $\delta_{m}(L):=\min _{S \in \mathrm{S}_{m}^{>0}(\mathbb{R}) \mid L_{S} \neq \emptyset} \operatorname{det} S$. Let $S_{m}(L):=\cup L_{S}$, where $S \in \mathrm{S}_{m}^{>0}(\mathbb{R})$ and $\operatorname{det} S=\delta_{m}(L)$. The finite set $S_{m}(L)$ is called the set of minimal $m$-sections of the lattice $L$.

In particular, $\delta_{1}(L)=\min (L)$. The minimal 1-sections are the lines supporting the minimal vectors of the lattice.

\section{Grassmannian designs and Rankin constants of lattices}

Beside the classical Hermite function $\gamma\left(=\gamma_{1}\right.$ in what follows), Rankin defined a collection of functions $\gamma_{m}$ associated with a lattice $L \subset \mathbb{R}^{n}$ :

$$
\gamma_{m}(L):=\delta_{m}(L) /(\operatorname{det} L)^{\frac{m}{n}}
$$

Thus, for $m=1, \gamma_{1}(L)$ is the classical Hermite invariant of $L$. As a function on the set of $n$-dimensional positive definite lattices, $\gamma_{m}$ is bounded, and the supremum, which actually is a maximum, is denoted by $\gamma_{m, n}$. In [13], a characterization of the local maxima of $\gamma_{m}$ was given.

Definition. (1) A lattice $L$ is called $m$-perfect if the endomorphisms $\operatorname{pr}_{p}$ when $p \in S_{m}(L)$ generate $\operatorname{End}^{s}(E)$

(2) A lattice $L$ is $m$-eutactic if there exist positive coefficients $\lambda_{p}$, $p \in S_{m}(L)$ such that $\sum_{p \in \mathcal{S}_{m}(L)} \lambda_{p} \mathrm{pr}_{p}=I d$.

(3) A lattice $L$ is called $m$-extreme, if $\gamma_{m}$ achieves a local maximum at $L$.

Theorem 3.1 ([13]). $L$ is $m$-extreme if and only if $L$ is both $m$-perfect and m-eutactic.

Theorem 3.2 ([29], [3]). If $S_{m}(L)$ is a 4-design in $\mathcal{G}_{m, n}$, then it is m-extreme, i.e. it achieves a local maximum of the Rankin function $\gamma_{m}$. 
Following B. Venkov, who calls strongly perfect a lattice for which $S(L)$ is a 4-design, we call $m$-strongly perfect a lattice $L$ for which $S_{m}(L)$ is a 4-design in $\mathcal{G}_{m, n}$. It is worth noticing that, since the number of classes of $m$-perfect lattices is finite, the number of classes of strongly $m$-perfect lattices is also finite.

Examples: The main sources of examples are the following:

- Small dimensional lattices gave the first examples of $m$-strongly lattices: in that case, it can be checked directly, using Theorem 2.1. It was natural to look among the strongly perfect lattices, which have been classified up to dimension $n \leq 12$ ([29], [22], [23]). These are: $A_{2}, D_{4}, E_{6}, E_{7}, E_{8}, K_{10}^{\prime}, K_{10}^{\prime}{ }^{*}, K_{12}$. They are $m$-strongly perfect for all $m$, except $K_{10}^{\prime}$, its dual, and $K_{12}$, which are only 1-strongly perfect.

- Extremal modular lattices. In that case, the spherical theta series of the lattices can be used to prove strong perfection. This argument generalizes in principle to $m>1$. Only for $m=2$ and the even unimodular case explicit calculations on the spaces of vector-valued Siegel modular forms show that certain families of lattices are 2strongly perfect, namely the extremal ones of dimension 32 and 48 (see [29], [5], [4]).

- Lattices with an automorphism group whose natural representation satisfies the criterion of Theorem 4.1 of the next section. This case leads to many examples (see Table 1), and to an infinite family of $m$-strongly perfect lattices: the sequence of the Barnes-Wall lattices, which will be discussed in section 5 .

\section{Orbits of finite subgroups of $\mathrm{O}\left(\mathbb{R}^{n}\right)$.}

A natural way to produce finite subsets of $\mathcal{G}_{m, n}$ is to take the orbit of a point under the action of a finite subgroup $G$ of $\mathrm{O}\left(\mathbb{R}^{n}\right)$. In [3], we prove a criterion on the representations of $G$ for these sets to be designs, which naturally extends a well-known criterion for the spherical designs.

Theorem 4.1 ([3]). Let $m_{0} \leq n / 2$. Let $G<\mathrm{O}\left(\mathbb{R}^{n}\right)$ be a finite group. The following conditions are equivalent:

- For all $m \leq m_{0}$ and all $p \in \mathcal{G}_{m, n}, G$.p is a $2 t$-design

- For all $\mu, 1 \leq \operatorname{deg}(\mu) \leq t, l(\mu) \leq m_{0},\left(V_{n}^{2 \mu}\right)^{G}=\{0\}$ 
Proof. We give here a simplified proof. Assume $\mathcal{D}=G . p$ is the orbit of $p \in \mathcal{G}_{m, n}$. Let $G_{p}$ be the stabilizer of $p$. Then,

$$
\begin{aligned}
\sum_{x \in \mathcal{D}} f(x) & =\frac{1}{\left|G_{p}\right|} \sum_{g \in G} f(g \cdot p) \\
& =\frac{1}{\left|G_{p}\right|} \sum_{g \in G}\left(g^{-1} \cdot f\right)(p) \\
& =\frac{|G|}{\left|G_{p}\right|}\left(\epsilon_{G} \cdot f\right)(p)
\end{aligned}
$$

where $\epsilon_{G}=\frac{1}{|G|} \sum_{g \in G} g$. The condition $\left(V_{n}^{2 \mu}\right)^{G}=\{0\}$ is equivalent to $\epsilon_{G}\left(V_{n}^{2 \mu}\right)=\{0\}$ which from previous equalities and the characteristic condition (2.3) lead to the statement.

Examples: It is well-known that the Weyl groups of irreducible root systems $W(R)$ acting on the space of homogeneous polynomials of degree 2 leave invariant only the quadratic form $x_{1}^{2}+x_{2}^{2}+\cdots+x_{n}^{2}$. Therefore, these groups give rise to 2-designs on all the Grassmannian spaces. Moreover, the property for the degree 4 holds also for $A_{2}, D_{4}, E_{6}, E_{7}$ and the degree 6 is fulfilled for $E_{8}$. It is easily checked directly on the groups; note that the partitions to be taken into account are not only (4) and (6) but also, when $n \geq 4(2,2),(4,2)$, and, when $n \geq 6,(2,2,2)$.

The group 2.Co $o_{1}$ has the required property for the degree 10, with no restriction on $m$.

Another interesting example is the sequence of real Clifford groups $\mathcal{C}_{k}$ which are subgroups of $O\left(\mathbb{R}^{2^{k}}\right)$, leading to 6-designs in all the Grassmannians. Next section considers this group and one subgroup of index 2 which is the automorphism group of the Barnes-Wall lattice.

When the previous theorem can be applied to the group of automorphisms of a lattice $L$, since obviously the sets $L_{S}$ are unions of orbits under the action of $\operatorname{Aut}(L)$, we obtain that all these sets are designs.

When the strength is equal to 4 , the possible partitions are (2), (4), $(2,2)$. We have investigated the behavior of $\operatorname{Aut}(L)$ for all the lattices $L$ of dimension $4 \leq n \leq 26$ which are known to be strongly perfect. The results are summarized in Table 1 , where only one lattice among $\left\{L, L^{*}\right\}$ appears, even when they are not similar lattices.

The following situations occur (encoded in the last column of the table): 
(1) $G=\operatorname{Aut}(L)$ satisfies $\left(V_{n}^{\mu}\right)^{G}=\{0\}$ for the three possible partitions (2), (4), (2,2). In that case, the sets $L_{S}$ are 4-designs for all $S$, and in particular $L$ is strongly $m$-perfect for all $m$. It holds also for any lattice with the same automorphism group, especially for the dual lattice.

(2) $G=\operatorname{Aut}(L)$ satisfies $\left(V_{n}^{\mu}\right)^{G}=\{0\}$ only for (2) and (4). We can only conclude that the sets $L_{m}:=\{x \in L \mid x \cdot x=m\}$, also called the layers of the lattice are 4-designs, as well as the layers of the dual lattice.

(3) $G=\operatorname{Aut}(L)$ does not satisfy $\left(V_{n}^{\mu}\right)^{G}=\{0\}$ for (2) and (4).

Moreover, one can ask if any of these lattices have an automorphism group holding the property of Theorem 4.1 for $t \geq 3$. It is well-known for the Leech lattice and $t=5$ (and not for $t=6$ ), and next section proves that the lattices $E_{8}$ and $\Lambda_{16}$ reache $t=3$. A direct calculation shows that the minimal vectors of $E_{8}$ and $\Lambda_{16}$ do not hold an 8-design, so $t=3$ is the maximum. The classification of the integral lattices of minimum $m \leq 5$ whose set of minimal vectors is a 6 -design, performed in [18], shows that the other lattices in this table cannot exceed $t=2$, except possibly the lattice $N_{16}$, and the lattices $O_{23}$ and $\Lambda_{23}$ (the lattice $O_{23}$ is missing in the list of lattices given in [18, Théorème], see the Erratum at: http://www.math.u-bordeaux1.fr/ martinet). A direct computation on the automorphism groups shows that $t=3$ is the maximum for $O_{23}$ and $\Lambda_{23}$, respectively $t=2$ for $N_{16}$.

The list of these lattices is taken from [29], with an additionnal lattice of dimension 26 which was pointed to me by J. Martinet (named T26 after [21]. The lattice $N_{26}$ appears in [21] as $B_{e i s_{26}}$ and $S_{6}(3) C_{3} .2$.)

We have kepted the notations of [29] for the names of the lattices, except of course for the last one. The determinant is given in the third column, in a form that reveals the structure of the discriminant group $L^{*} / L$. The automorphism group is given in the fifth column, with the notations of [19], [21] when available. In [29] and [21] more informations on these lattices are given.

The condition on $\left(V_{n}^{\mu}\right)^{G}$ is checked using the Schur polynomials associated with $\mu$.

A completely different reason for the existence of spherical designs in lattices is often given by the theory of modular forms (see [29], [5]). Among the list of Table 1, only the 21-dimensional lattice escapes from both the group theory argument and the modular forms argument. It is worth pointing out that it is the only one of which the dual lattice does not have a 4 -spherical design on its minimal vectors. Of course, it is expected that the situation is completely different when the dimension grows, and the above list is anyway complete only up to the dimension 12 . 
TABLE 1

\begin{tabular}{|c|c|c|c|c|c|}
\hline $\operatorname{dim}$ & name & det & $\min$ & $\mathrm{G}$ & case \\
\hline 4 & $D_{4}$ & 4 & 2 & $W\left(F_{4}\right)$ & (1) \\
\hline 6 & $E_{6}$ & 3 & 2 & $2 \times W\left(E_{6}\right)$ & (1) \\
\hline 7 & $E_{7}$ & 2 & 2 & $W\left(E_{7}\right)$ & (1) \\
\hline 8 & $E_{8}$ & 1 & 2 & $W\left(E_{8}\right)$ & (1) \\
\hline 10 & $K_{10}^{\prime}$ & $6^{2} \cdot 3^{3}$ & 4 & $(6 \times S U(4,2)): 2$ & $(2)$ \\
\hline 12 & $K_{12}$ & $3^{6}$ & 4 & $6 . S U_{4}(3) .2^{2}$ & $(2)$ \\
\hline 14 & $Q_{14}$ & $3^{7}$ & 4 & $2 \times G_{2}(3)$ & (1) \\
\hline 16 & $\Lambda_{16}$ & $2^{8}$ & 4 & $2_{+}^{9} \Omega^{+}(8,2)$ & (1) \\
\hline- & $O_{16}$ & $2^{6}$ & 3 & $D_{8}^{4} \cdot S_{6}(2)$ & $(1)$ \\
\hline- & $N_{16}$ & $5^{8}$ & 6 & $2 . A l t_{10}$ & $(2)$ \\
\hline 18 & $K_{18}^{\prime}$ & $3^{5}$ & 4 & $\left(2 \times 3^{1+4}: S p_{4}(3)\right) .2$ & $(2)$ \\
\hline 20 & $N_{20}$ & $2^{10}$ & 4 & $\left(S U_{5}(2) \times S L_{2}(3)\right) .2$ & $(2)$ \\
\hline- & $N_{20}^{\prime}$ & - & - & $2 . M_{12} .2$ & $(2)$ \\
\hline- & $N_{20}^{\prime \prime}$ & - & - & $H S_{20}$ & $(3)$ \\
\hline 21 & $K_{21}^{\prime}$ & $12 \cdot 3$ & 4 & $2^{11} \cdot 3^{6} \cdot 5.7$ & (3) \\
\hline 22 & $\mathrm{O}_{22}$ & 3 & 3 & {$\left[\operatorname{Aut}\left(\Lambda_{22}\right): \operatorname{Aut}\left(O_{22}\right)\right]=3$} & (1) \\
\hline - & $\Lambda_{22}$ & $6 \cdot 2$ & 4 & $\left(2 \times P S U_{6}(2)\right) \cdot S_{3}$ & (1) \\
\hline - & $\Lambda_{22}[2]$ & $6 \cdot 2^{19}$ & 6 & - & - \\
\hline- & $M_{22}$ & 15 & 4 & $(2 \times M c L) .2$ & (1) \\
\hline - & $M_{22}[5]$ & $15 \cdot 3^{20}$ & 10 & - & - \\
\hline 23 & $\mathrm{O}_{23}$ & 1 & 3 & $2 \times \mathrm{CO}_{2}$ & (1) \\
\hline - & $\Lambda_{23}$ & 4 & 4 & - & - \\
\hline- & $M_{23}$ & 6 & 4 & $2 \times \mathrm{CO}_{3}$ & (1) \\
\hline- & $M_{23}[2]$ & $6 \cdot 3^{21}$ & 10 & - & - \\
\hline 24 & $\Lambda_{24}$ & 1 & 4 & $2 . C O_{1}$ & (1) \\
\hline 24 & $N_{24}$ & $3^{12}$ & 6 & $S L_{2}(13) \circ S L_{2}(3)$ & $(3)$ \\
\hline 26 & $N_{26}$ & $3^{13}$ & 6 & $S_{6}(3) C_{3} .2$ & (3) \\
\hline 26 & $T_{26}$ & 3 & 4 & $3 D_{4}(2): 3$ & (3) \\
\hline
\end{tabular}

\section{The group $\operatorname{Aut}\left(B W_{n}\right)$}

In this section we study the tensor invariants of the automorphism group of the Barnes-Wall lattices. We shall make use of the methods and results developed in [20]. Let us recall from [20] some facts about the Clifford groups $\mathcal{C}_{k}$ and the Barnes-Wall lattices.

We set $n=2^{k}$. The real space $\mathbb{R}^{n}$ is endowed with an orthonormal basis $\left(e_{u}\right)_{u \in F_{2}^{k}}$ indexed by the elements of $\mathbb{F}_{2}^{k}$. 
The Barnes-Wall lattice $B W_{n} \subset \mathbb{R}^{n}$ is the lattice defined by:

$$
B W_{n}=<2^{\left\lfloor\frac{k-d+1}{2}\right\rfloor} \sum_{u \in U} e_{u}, U>_{\mathbb{Z}}
$$

where $U$ runs over all affine subspaces of $F_{2}^{k}$, and $d=\operatorname{dim}(U)$.

The first lattices of the sequence are well-known: $B W_{4} \simeq D_{4}, B W_{8} \simeq E_{8}$, $B W_{16} \simeq \Lambda_{16}$ the laminated lattice of the dimension 16 . Suitably rescaled, $\min \left(B W_{n}\right)=2^{\left\lfloor\frac{k}{2}\right\rfloor}$, and $B W_{n}$ is even unimodular when $k \equiv 1 \bmod 2$, respectively 2-modular when $k \equiv 0 \bmod 2$.

Bolt, Room and Wall ([9], [10], [8]) and later Broué-Enguehard [7] described $\operatorname{Aut}\left(B W_{n}\right)$. When $n \neq 8$, it is a subgroup of index 2 in the Clifford group $\mathcal{C}_{k}$ which we describe now.

The extra-special 2-group $2_{+}^{1+2 k}$ has a representation $E$ in $\mathbb{R}^{n}$ : if

$$
\begin{gathered}
X(a): e_{u} \rightarrow e_{u+a} \text { and } Y(b): e_{u} \rightarrow(-1)^{b \cdot u} e_{u}, \\
E=<-I, X(a), Y(b) \mid a, b \in \mathbb{F}_{2}^{k}>.
\end{gathered}
$$

Definition. The Clifford group $\mathcal{C}_{k}$ is the normalizer in $O\left(\mathbb{R}^{n}\right)$ of $E$.

Since $q(x):=x^{2}$ defines a quadratic form on $E / Z(E) \simeq \mathbb{F}_{2}^{2 k}$, non degenerate and of maximal Witt index, and since $\mathcal{C}_{k}$ acts on $E$ (by conjugation) preserving $q$, it induces a subgroup of $O^{+}(2 k, 2)$. It turns out that the whole $O^{+}(2 k, 2)$ is realized, yielding the isomorphism:

$$
\mathcal{C}_{k} \simeq 2_{+}^{1+2 k} . O^{+}(2 k, 2)
$$

The group $O^{+}(2 k, 2)$ has a unique subgroup of index $2, \Omega^{+}(2 k, 2)$. Its parabolic subgroups are the stabilizers in $\Omega^{+}(2 k, 2)$ of totally isotropic subspaces; they are maximal in $\Omega^{+}(2 k, 2)$. Let $P(2 k, 2)$ be the one associated with the image in $\mathbb{F}_{2}^{2 k}$ of $\langle \pm X(a)| a \in \mathbb{F}_{2}^{k}>$.

According to [20], the following transformations are explicit generators of the group $\mathcal{C}_{k}$ :

(1) Diagonal transformations: $e_{u} \rightarrow(-1)^{q(u)} e_{u}$, where $q$ is any binary quadratic form, and $-I$.

(2) Permutation transformations: $e_{u} \rightarrow e_{\phi(u)}$, where $\phi \in A G L(k, 2)$.

(3) $H:=h \otimes I_{2} \otimes \cdots \otimes I_{2}, h=\frac{1}{\sqrt{2}}\left[\begin{array}{cc}1 & 1 \\ 1 & -1\end{array}\right]$ (here $\mathbb{R}^{n}$ and $\left(\mathbb{R}^{2}\right)^{\otimes k}$ are identified in an obvious way).

Straightforward calculations show that these elements normalize $E$. Moreover, the induced action of the elements of the first and second type on $\mathbb{F}_{2}^{2 k}$ is given by the respective matrices $\left[\begin{array}{ll}1 & b \\ 0 & 1\end{array}\right]$ where $b$ is the symplectic 
matrix associated with $q$, and $\left[\begin{array}{cc}\phi & 0 \\ 0 & \phi^{-t r}\end{array}\right]$ where $\phi \in G L(2, k)$. The group generated by these transformations on $\mathbb{F}_{2}^{2 k}$ is the parabolic group $P(2 k, 2)$.

The element $H_{2}:=h \otimes h \otimes I_{2} \otimes \cdots \otimes I_{2}$ has rational entries. The subgroup $\mathcal{G}_{k}$ of $\mathcal{C}_{k}$ generated by the elements of the first and second type, and $H_{2}$, generate a subgroup of $\Omega^{+}(2 k, 2)$, containing $P(2 k, 2)$, hence equal to $\Omega^{+}(2 k, 2)$. It follows that $\mathcal{G}_{k}$ has index 2 in $\mathcal{C}_{k}$ and is rational; hence it is the automorphism group of $B W_{n}$ (see [20]).

The polynomial invariants of $\mathcal{C}_{k}$ are described, first by B. Runge ([24], [25], [26]), then with a different proof by G. Nebe, E. Rains, N.J.A. Sloane ([20], in terms of self-dual binary codes. As a consequence, the first non trivial invariant occurs for the degree 8 , associated with the first non trivial self-dual binary code which is the $[8,4,4]$ Hamming code. We extend here this result to the subgroup $\mathcal{G}_{k}$.

Theorem 5.1. If $k \geq 3$ and $d \leq 6$, then

$$
\left(V^{\otimes d}\right)^{\mathcal{G}_{k}}=\left(V^{\otimes d}\right)^{\mathcal{C}_{k}}=\left(V^{\otimes d}\right)^{O(V)} .
$$

Corollary 5.1. The orbits of $\operatorname{Aut}\left(B W_{n}\right)$ on $\mathcal{G}_{m, n}$ are 6-designs. In particular, the sets $\left(B W_{n}\right)_{S}$ are 6-designs and the lattice $B W_{n}$ is strongly $m$-perfect for all $m$.

Remark. - Theorem 5.1 shows more than what is needed for the Grassmannian design property, since $V^{\otimes 6}$ contains the representations associated with arbitrary partitions of degree lower or equal to 6 .

- The fact that the set of minimal vectors is a 6-spherical design was already proved by direct calculation by Boris Venkov ([29]).

Proof. The argument in [20] extends straightforwardly to the tensor invariants of $\mathcal{C}_{k}$. Let $V:=\mathbb{R}^{n}$. To a binary code $C$ of length $d$, is associated a tensor enumerator $T_{C}^{(k)} \in V^{\otimes d}$. To a $k$-tuple $\left(w_{1}, \ldots, w_{k}\right)$ of codewords, we associate a $k \times d$ matrix which rows are the words $w_{1}, \ldots, w_{k}$. Let $u_{1}, \ldots, u_{d}$ be the $d$ columns of this matrix. Then:

$$
T_{C}^{(k)}:=\sum_{\left(w_{1}, \ldots, w_{k}\right) \in C^{k}} e_{u_{1}} \otimes \cdots \otimes e_{u_{d}}
$$

where

The usual (generalized) weight enumerator $W_{C}^{(k)}$ is obtained by the symmetrization $V^{\otimes d} \rightarrow \operatorname{Sym}^{d}(V)$. For the same reasons, when $C$ is self-dual, $T_{C}^{(k)}$ is invariant under the action of $\mathcal{C}_{k}$. A straightforward generalization of the proof in [20] of the fact that the invariants of $\mathcal{C}_{k}$ on $\operatorname{Sym}^{d}(V)$ are exactly spanned by the polynomials $W_{C}^{(k)}$ when $C=C^{\perp}$ shows that the 
invariants of $\mathcal{C}_{k}$ on $V^{\otimes d}$ are spanned by the $T_{C}^{(k)}$ when $C=C^{\perp}$. To determine the invariants of $\mathcal{G}_{k}$, we follow the same steps as in [20]: the first is the description of $\left(V^{\otimes d}\right)^{\mathcal{P}_{k}}$, which we recall in next lemma.

Lemma 5.1 ([20], Theorem 4.6). The space $\left(V^{\otimes d}\right)^{\mathcal{P}_{k}}$ is generated by the $T_{C}^{(k)}$ where $C$ runs over the binary codes of length $d$ such that $1 \subset C \subset C^{\perp}$ and $\operatorname{dim}(C) \leq k+1$.

The second calculates $\epsilon_{\mathcal{P}_{k}} H_{2}$ as a linear combination of the $T_{C}^{(k)}$ associated with binary codes satisfying $1 \subset C \subset C^{\perp}$ (which obviously belong to $\left(V^{\otimes d}\right)^{\mathcal{P}_{k}}$; only those with $\operatorname{dim}(C) \leq k+1$ are linearly independent).

Lemma 5.2. Let $C$ be a binary code of length $d$ such that $1 \subset C \subset C^{\perp}$ and $\operatorname{dim}(C) \leq k+1$. Let $r:=d / 2-\operatorname{dim}(C)$.

$$
\left(\epsilon_{\mathcal{P}_{k}} H_{2}\right) \cdot T_{C}^{(k)}=a_{1} T_{C}^{(k)}+a_{2} \sum_{\substack{C^{\prime} \subset C^{\prime \perp} \\ C \subset C^{\prime},\left[C^{\prime}: C\right]=2}} T_{C^{\prime}}^{(k)}+a_{4} \sum_{\substack{C^{\prime} \subset C^{\prime} \\ C \subset C^{\prime},\left[C^{\prime}: C\right]=4}} T_{C^{\prime}}^{(k)}
$$

where

$$
\left\{\begin{array}{l}
a_{1}=2^{-2 r}\left(1+2 \frac{\left(2^{2 r}-1\right)\left(2^{2 r-2}-1\right)}{\left.2^{k}-1\right)\left(2^{k-1}-1\right)}-3 \frac{2^{2 r}-1}{2^{k}-1}\right) \\
a_{2}=\frac{3.2^{-2 r}}{2^{k}-1}\left(1-\frac{2^{2 r-2}-1}{2^{k-1}-1}\right) \\
a_{4}=\frac{3.2^{-2 r}}{\left(2^{k}-1\right)\left(2^{k-1}-1\right)}
\end{array}\right.
$$

Moreover, $a_{1}=1$ if and only if $r=0$ or $r=k$.

Proof. Let $\mu\left(w_{1}, \ldots, w_{k}\right):=e_{u_{1}} \otimes \cdots \otimes e_{u_{d}}$. We have (as a consequence of the Poisson summation formula)

$$
H_{2} T_{C}^{(k)}=2^{-2 r} \sum_{\substack{w_{1}, w_{2} \in C^{\perp} \\ w_{3}, \ldots, w_{k} \in C}} \mu\left(w_{1}, \ldots, w_{k}\right)
$$

As a consequence of the change from $\mathrm{H}$ to $\mathrm{H}_{2}$, not only the first, but also the second vector is allowed to be in $C^{\perp}$. Therefore, by the same argument as in [20], there exists coefficients $a_{1}, a_{2}, a_{4}$ (depending on $r$ and $k$ ) such that

$$
\epsilon_{\mathcal{P}_{k}} H_{2} T_{C}^{(k)}=a_{1} T_{C}^{(k)}+a_{2} \sum_{\substack{C^{\prime} \subset C^{\prime} \perp \\ C \subset C^{\prime},\left[C^{\prime}: C\right]=2}} T_{C^{\prime}}^{(k)}+a_{4} \sum_{\substack{C^{\prime} \subset \subset C^{\prime} \\ C \subset C^{\prime},\left[C^{\prime}: C\right]=4}} T_{C^{\prime}}^{(k)}
$$

and we are left with the computation of these coefficients. Let $<,>$ denote the scalar product induced on $V^{\otimes d}$ by the Euclidean structure on $V$. For any codes $C, D$, with $\mathbf{1} \subset C \subset D \subset D^{\perp} \subset C^{\perp}$, we have:

$$
<T_{C}^{(k)}, T_{D}^{(k)}>=|C|^{k}
$$


and

$$
<\left(\epsilon_{\mathcal{P}_{k}} H_{2}\right) \cdot T_{C}^{(k)}, T_{D}^{(k)}>=<H_{2} \cdot T_{C}^{(k)}, T_{D}^{(k)}>=2^{-2 r}[D: C]^{2}|C|^{k} .
$$

Let $n_{2}^{r}$, respectively $n_{4}^{r}$ be the number of self-orthogonal codes containing $C$ to index 2, respectively 4 . Obviously, $n_{2}^{r}$ equals the number of isotropic lines in the symplectic space $C^{\perp} / C$ of dimension $2 r$, and $n_{4}^{r}$ equals the number of totally isotropic planes in $C^{\perp} / C$. Therefore, $n_{2}^{r}=2^{2 r}-1$ and $n_{4}^{r}=\left(2^{2 r}-1\right)\left(2^{2 r-2}-1\right) / 3$. Taking the scalar product of equation (5.1) with $T_{D}^{(k)}$, successively for $D=C$, then for a self-orthogonal code containing $C$ to index 2 and 4 , we obtain the three equations (after having divided by $\left.|C|^{k}\right)$ :

$$
\begin{aligned}
2^{-2 r}= & a_{1}+a_{2} n_{2}^{r}+a_{4} n_{4}^{r} \\
2^{-2 r} .4= & a_{1}+a_{2} \cdot 2^{k}+a_{2}\left(n_{2}^{r}-1\right)+a_{4} n_{2}^{r-1} \cdot 2^{k}+a_{4}\left(n_{4}^{r}-n_{2}^{r-1}\right) \\
2^{-2 r} .16= & a_{1}+a_{2} \cdot 3 \cdot 2^{k}+a_{2}\left(n_{2}^{r}-3\right) \\
& +a_{4} \cdot 4^{k}+a_{4}\left(3 n_{2}^{r-1}-3\right) \cdot 2^{k}+a_{4}\left(n_{4}^{r}-3 n_{2}^{r-1}+2\right)
\end{aligned}
$$

which lead to the expressions of Theorem 5.1 .

We end the proof of the theorem in the same way as in [20]. We have $\left(V^{\otimes d}\right)^{\mathcal{G}_{k}}=\operatorname{ker}\left(\epsilon_{\mathcal{P}_{k}} H_{2}-I\right) \cap\left(V^{\otimes d}\right)^{\mathcal{P}_{k}}$. From Lemma 5.2, when the elements $T_{C}^{(k)}$ are ordered by increasing $\operatorname{dim}(C)$, the matrix of the transformation $\epsilon_{\mathcal{P}_{k}} H_{2}$ is upper triangular. If $k \leq 3$ and $d \leq 6$, the only diagonal coefficients which are equal to 1 correspond to $C=C^{\perp}$ and we can conclude by [20], Lemma 4.8

Remark. Of course, for arbitrary degree $d$, the group $\mathcal{G}_{k}$ has more invariants than $\mathcal{C}_{k}$. For $k=2$ and $d=6$, we have $a_{1}=1$ for $r=2$, i.e. for the code $C=1$. The element

$$
T_{\mathbf{1}}^{(2)}-\frac{1}{12} \sum_{\substack{1 \subset C \subset C^{\perp} \\ \operatorname{dim}(C)=2}} T_{C}^{(2)}
$$

is the unique degree 6 additional invariant under $\mathcal{G}_{2}$.

For $k=3$ and $d=8$, the situation is the same, with

$$
T_{1}^{(3)}-\frac{1}{40} \sum_{\substack{1 \subset C \subset C^{\perp} \\ \operatorname{dim}(C)=2}} T_{C}^{(3)}+\frac{1}{480} \sum_{\substack{1 \subset C \subset C^{\perp} \\ \operatorname{dim}(C)=3}} T_{C}^{(3)}
$$


as an invariant of degree 8 . The Molien series confirms that the degree 8 polynomial invariant space has dimension 3 , spanned by the two classes of self-dual codes and this one.

\section{Other Grassmannian designs}

When a group $G$ is known to fulfill the conditions of Theorem 4.1, among its orbits the most interesting ones are the ones shorter than the "generic" ones, i.e. the ones with a non trivial isotropic group. In general, it is not easy to describe these orbits. In the case of the Clifford group $\mathcal{C}_{k}$, some of these orbits are described in a very explicit way in [11], in view of the construction of Grassmannian codes for the chordal distance. We next discuss under which conditions certain smaller subsets of these sets remain to be 6-designs or 4-designs. More precisely, we prove that it depends on a similar condition of design associated with the underlying finite geometry.

6.1. The construction. The alluded construction is the following. Let $S \subset \mathbb{F}_{2}^{2 k}$ be a totally isotropic subspace of dimension $k-s$. The preimage $\tilde{S}$ of $S$ in $E$ is an abelian g:oup, 2-elementary. (The identification between $\mathbb{F}_{2}^{2 k}$ and $E /\{ \pm 1\}$ is still the same, sending $X(a) Y(b)$ to $(a, b))$, It decomposes the space $V=\mathbb{R}^{n}$ into $2^{k-s}$ irreducible subspaces of dimension $2^{s}$, which are pairwise orthogonal. Let $\mathcal{D}_{S} \subset \mathcal{G}_{2^{s}, 2^{k}}$ be the set of these $2^{k-s}$ subspaces.

$\mathrm{M}, \mathrm{rr}$ generally, if $\Sigma$ is a set of isotropic subspaces of the same dimension $k-s$, we set

$$
\mathcal{D}_{\Sigma}:=\cup_{S \in \Sigma} \mathcal{D}_{S} \subset \mathcal{G}_{2^{s}, 2^{k}} .
$$

Example: We can take $\Sigma$ to be the whole set of totally isotropic subspaces of fixed dimension $k-s$. In that case, $\Sigma$ is a single orbit under $O^{+}(2 k, 2)$, and $\mathcal{D}_{\Sigma}$ is a single orbit under $\mathcal{C}_{k}$. Therefore it is a 6-design. Note that, when $s=0, \Sigma$ splits into two orbits under the action of $\Omega^{+}(2 k, 2)$; the set of lines corresponding to one orbit is the set of lines supporting the minimal vectors of $B W_{n}, n=2^{k}$.

Of course, we are interested in the smallest possible sets, and the above example is the largest one. A natural question is then: which conditions should $\Sigma$ satisfy, so that $\mathcal{D}_{\Sigma}$ is a design? How small can we take $\Sigma$ ? To answer these questions, we need two more ingredients: another criterion for Grassmannian designs, and the notion of designs on the spaces of totally isotropic subspaces of fixed dimension. 
6.2. A new criterion. Let $\mathcal{D} \subset \mathcal{G}_{m, n}$. Let $\sigma:=y_{1}+y_{2}+\cdots+y_{m}$.

Theorem 6.1. For all $m, n$, and $t$, there exists a constant $c_{m, n}(2 t)$ such that

(1) For all $\mathcal{D} \subset \mathcal{G}_{m, n}, \frac{1}{|\mathcal{D}|^{2}} \sum_{p, p^{\prime} \in \mathcal{D}} \sigma\left(p, p^{\prime}\right)^{t} \geq c_{m, n}(2 t)$.

(2) $\mathcal{D}$ is a $2 t$-design if and only if $\frac{1}{|\mathcal{D}|^{2}} \sum_{p, p^{\prime} \in \mathcal{D}} \sigma\left(p, p^{\prime}\right)^{t}=c_{m, n}(2 t)$.

Proof. From the defining property of Grassmannian designs (Definition 2.1 ), since $\sigma^{t}$ has degree $t$ in the variables $y_{1}, \ldots, y_{m}$, if $\mathcal{D}$ is a $2 t$-design,

$$
\frac{1}{|\mathcal{D}|^{2}} \sum_{p, p^{\prime} \in \mathcal{D}} \sigma\left(p, p^{\prime}\right)^{t}=\int_{[0,1]^{m}} \sigma^{t} d \mu\left(y_{1}, \ldots, y_{m}\right)
$$

We set $c_{m, n}(2 t):=\int_{[0,1]^{m}} \sigma^{t} d \mu\left(y_{1}, \ldots, y_{m}\right)$.

Lemma 6.1. There exists positive coefficients $\lambda_{t, \mu}>0$ such that:

$$
\sigma^{t}=\sum_{\mu, \operatorname{deg}(\mu) \leq t} \lambda_{t, \mu} P_{\mu}
$$

Proof. For $t=1$, we have $\sigma=\frac{m(n-m)}{n} P_{(1)}+\frac{m^{2}}{n}$.

For $t>1$, we proceed by induction. Let us assume first that $\operatorname{deg}(\mu)<t$. We have

$$
\begin{aligned}
{\left[\sigma^{t}, P_{\mu}\right] } & =\left[\sigma^{t-1}, \sigma P_{\mu}\right] \\
& =\left[\sigma^{t-1},\left(\frac{m(n-m)}{n} P_{(1)}+\frac{m^{2}}{n}\right) P_{\mu}\right]
\end{aligned}
$$

We know that $P_{(1)} P_{\mu}$ is a linear combination with non negative coefficients of the $P_{\kappa}$ ([1, Lemma 2]). By induction, we obtain

$$
\begin{aligned}
{\left[\sigma^{t}, P_{\mu}\right] } & \geq\left[\sigma^{t-1}, \frac{m^{2}}{n} P_{\mu}\right] \\
& >0(\text { if } \operatorname{deg}(\mu)<t) .
\end{aligned}
$$

If $\operatorname{deg}(\mu)=t$, the second term of the first inequality is zero. We need more information on the expression $\sigma P_{\mu}$ on the $P_{\kappa}$. The analogue of the "three-term relation" for orthogonal polynomials in one variable gives (see [2]):

$$
\sigma P_{\mu}=\sum_{\operatorname{deg}(\kappa)=k+1} A_{k}[\mu, \kappa] P_{\kappa}+\sum_{\operatorname{deg}(\kappa)=k} B_{k}[\mu, \kappa] P_{\kappa}+\sum_{\operatorname{deg}(\kappa)=k-1} C_{k}[\mu, \kappa] P_{\kappa}
$$

where $k=\operatorname{deg}(\mu)$. Moreover, $C_{k}[\mu, \kappa]\left[P_{\kappa}, P_{\kappa}\right]=A_{k-1}[\kappa, \mu]\left[P_{\mu}, P_{\mu}\right]$ is zero unless $\mu$ is obtained from $\kappa$ by the increase of one of its parts by one, 
in which case $A_{k-1}[\kappa, \mu]>0([2])$. In $\left[\sigma^{t}, P_{\mu}\right]=\left[\sigma^{t-1}, \sigma P_{\mu}\right]$ only those terms (and at least one) give a contribution, so, by induction, we obtain the desired property.

Since $c_{m, n}(2 t)=\left[\sigma^{t}, 1\right]=\lambda_{t, 0}$, and from the design criterion and the positivity condition of Theorem 2.1, the proof of Theorem 6.1 is completed (note that it is crucial than none of the $\lambda_{t, \mu}$ is equal to zero).

Remark. This criterion is analogous to [29, Théorème 8.1], and similar versions exist in principle for any notion of design. We shall come across a similar criterion for the designs of totally isotropic spaces.

Remark. It is not apparently easy to calculate $c_{m, n}(2 t)$ by the integration formula $c_{m, n}(2 t):=\int_{[0,1]^{m}} \sigma^{t} d \mu\left(y_{1}, \ldots, y_{m}\right)$. It is worth noticing that, since $c_{m, n}(2 t)=\left[\sigma^{t}, 1\right]=\lambda_{t, 0}$, it becomes easy once one has calculated explicitly the polynomials $P_{\mu}$ for $\operatorname{deg}(\mu) \leq t$. For example, we obtain from $\S 2.1$,

$$
\begin{aligned}
& c_{m, n}(2)=\frac{m^{2}}{n} \\
& c_{m, n}(4)=\frac{m^{2}}{3 n}\left(\frac{2(m-1)^{2}}{n-1}+\frac{(m+2)^{2}}{n+2}\right)
\end{aligned}
$$

and, using $\left[\sigma^{3}, 1\right]=\left[\sigma^{2}, \sigma\right]$ and $\left[P_{(1)}, P_{(1)}\right]=\operatorname{dim}\left(V_{n}^{(2)}\right)^{-1}=\frac{2}{(n-1)(n+2)}$, we can even calculate

$$
\begin{aligned}
c_{m, n}(6)=\frac{m^{2}}{3 n}\left(\frac{(m-1)^{2}(m+2)^{2}}{(n-1)(n+2)}\left(\frac{2 n}{n-2}+\frac{n+3}{n+4}\right)\right. \\
\left.-8 \frac{m(m-1)^{2}}{(n-1)(n-2)}+\frac{(m+2)^{2}(2 m+3)}{(n+2)(n+4)}\right)
\end{aligned}
$$

6.3. The space of totally isotropic subspaces. Let $X_{w}$ be the set of totally isotropic subspaces of dimension $w \leq k$ of the quadratic space $\left(\mathbb{F}_{2}^{2 k}, q\right)$. The group $G:=O^{+}(2 k, 2)$ acts transitively on $X_{w}$; the stabilizer of an element is a maximal parabolic subgroup $P_{w}$. The orbits of $G$ on pairs of elements $\left(S, S^{\prime}\right)$ (also called orbitals) are investigated in [30]; they are characterized by two quantities: $\operatorname{dim}\left(S \cap S^{\prime}\right)$ and $\operatorname{dim}\left(S \cap S^{\prime \perp}\right)$. Since $\operatorname{dim}\left(S \cap S^{\perp}\right)=\operatorname{dim}\left(S^{\perp} \cap S^{\prime}\right)$, they are symmetric. In the special case $w=k$ of the maximal totally isotropic subspaces, $S=S^{\perp}$ and one value is enough, giving to $X_{w}$ the structure of a 2-point homogeneous space (for the distance $\left.d\left(S, S^{\prime}\right)=k-\operatorname{dim}\left(S \cap S^{\prime}\right)\right)$. 
The space $L\left(X_{w}\right)$ of complex valued functions on $X_{w}$ decomposes, under the action of $G$, into irreducible subspaces with multiplicities equal to one; to each subspace is associated a unique zonal function. This decomposition, and the corresponding zonal functions, are computed in [27], [28] (in [28], the general case of Chevalley groups over $\mathbb{F}_{q}$ is treated; it is assumed that the characteristic is different from 2, although the situation would be completely analogous. In [28], the case $w=k$ is treated in full generality). We only need here the general form of this decomposition ([27, Theorem $6.23])$ :

$$
L\left(X_{w}\right)=\oplus_{(m, r) \in I} V_{m, r}
$$

where $I:=\{(m, r) \mid 0 \leq m \leq w, 0 \leq r \leq m \wedge(k-w)\}$. If $y:=\operatorname{dim}\left(S \cap S^{\prime}\right)$, $x+y:=\operatorname{dim}\left(S \cap S^{\prime \perp}\right)$, the corresponding zonal (spherical) function $G_{m, r}$ is a polynomial in $2^{x}, 2^{y}$ and of degree $m$ in $2^{y}$. Note that $2^{y}=\left|S \cap S^{\prime}\right|$. When $w=k$, these polynomials are polynomials in one variable, and identified as $q$-Krawtchouk polynomials. In that case, the $t$-designs are defined in the usual way (see [15]).

Theorem 6.2. There exists constants $d_{w, k}(t)$ such that:

(1) For all $\Sigma \subset X_{w}, \frac{1}{|\Sigma|^{2}} \sum_{S, S^{\prime} \in \Sigma}\left|S \cap S^{\prime}\right|^{t} \geq d_{w, k}(t)$

(2) When $w=k$, equality holds if and only if $\Sigma$ is a $t$-design.

Remark. When $w<k$, the interpretation of the case of equality in terms of designs is not so clear. Since the irreducible spaces require a double index, the notion of $t$-designs itself is not so clear, although the most natural one would be, like in the case of the non-binary Johnson scheme, to require orthogonality with $\oplus V_{m, r}$, where $(m, r) \in\{(m, r) \mid m \leq t\}$. Then, one would need to look carefully at the positivity of the coefficients of the expansion of $\left(2^{y}\right)^{t}$ on the $G_{m, r}$. In the case $w=k$, the positivity is guaranteed, thanks to the three-terms relation, whose coefficients are the intersection numbers of the association scheme (see [6, II.2(2.1), III.1(1.2)]).

Here, the computation of the numbers $d_{w, k}(t)$ is easy, since they come from the constant term: $d_{w, k}(t)=\left[y^{t}, 1\right]$. So,

$$
\begin{aligned}
d_{w, k}(t) & =\frac{1}{\left|X_{w}\right|^{2}} \sum_{S, S^{\prime} \in X_{w}}\left|S \cap S^{\prime}\right|^{t} \\
& =\frac{1}{\left|X_{w}\right|^{2}} \sum_{x, y}|\operatorname{Orb}(x, y)|\left(2^{y}\right)^{t}
\end{aligned}
$$

where $\operatorname{Orb}(x, y)$ is the orbital associated with the values $(x, y)$; its cardinality is calculated in [30, Theorem 5.5]. 


\subsection{When is $\mathcal{D}_{\Sigma}$ a design?}

Theorem 6.3. Let $\mathcal{D}_{\Sigma}$ be defined as in (6.1).

(1) $\mathcal{D}_{\Sigma}$ is always a 2-design.

(2) For $t=2$ and $t=3, \mathcal{D}_{\Sigma}$ is a $2 t$-design if and only if $\Sigma$ satisfies the equality:

$$
\frac{1}{|\Sigma|^{2}} \sum_{S, S^{\prime} \in \Sigma}\left|S \cap S^{\prime}\right|^{t-1}=d_{w, k}(t-1) .
$$

Proof. We calculate $\frac{1}{\left|\mathcal{D}_{\Sigma}\right|^{2}} \sum_{p, p^{\prime} \in \mathcal{D}_{\Sigma}} \sigma\left(p, p^{\prime}\right)^{t}$. By the construction,

$$
\frac{1}{\left|\mathcal{D}_{\Sigma}\right|^{2}} \sum_{p, p^{\prime} \in \mathcal{D}_{\Sigma}} \sigma\left(p, p^{\prime}\right)^{t}=\frac{1}{2^{2(k-s)}|\Sigma|^{2}} \sum_{S, S^{\prime} \in \Sigma}\left(\sum_{p \in \mathcal{D}_{S}, p^{\prime} \in \mathcal{D}_{S^{\prime}}} \sigma\left(p, p^{\prime}\right)^{t}\right) .
$$

Let $\operatorname{dim}\left(S \cap S^{\prime}\right):=k-u$. The $2^{k-u}$ irreducible subspaces associated with $S \tilde{\cap} S^{\prime}$ are obtained from the $2^{k-s}$ ones associated with $S$, by summing together $2^{u-s}$ of them. These ones are precisely the ones on which the corresponding characters of $\tilde{S}$ and $\tilde{S}^{\prime}$ coincide on $S \tilde{\cap} S^{\prime}$. According to $[11,(9)]$, if $p \in \mathcal{D}_{S}$ and $p^{\prime} \in \mathcal{D}_{S^{\prime}}$ are contained in the same irreducible subspaces associated with $S \tilde{\cap} S^{\prime}$,

$$
\sigma\left(p, p^{\prime}\right)=2^{k} \frac{\left|S \cap S^{\prime}\right|}{|S|\left|S^{\prime}\right|}=2^{2 s-u}
$$

and it holds for $\left(2^{u-s}\right)^{2}$ pairs $\left(p, p^{\prime}\right)$. Otherwise, $\sigma\left(p, p^{\prime}\right)=0$, except if $p=p^{\prime}$ of course.

All together, we obtain

$$
\frac{1}{\left|\mathcal{D}_{\Sigma}\right|^{2}} \sum_{p, p^{\prime} \in \mathcal{D}_{\Sigma}} \sigma\left(p, p^{\prime}\right)^{t}=2^{(2 s-k) t} \frac{1}{|\Sigma|^{2}} \sum_{S, S^{\prime} \in \Sigma}\left|S \cap S^{\prime}\right|^{t-1} .
$$

From Theorem 6.1 , we obtain that $\mathcal{D}_{\Sigma}$ is a $2 t$-design if and only if

$$
\frac{1}{|\Sigma|^{2}} \sum_{S, S^{\prime} \in \Sigma}\left|S \cap S^{\prime}\right|^{t-1}=2^{-(2 s-k) t} c_{2^{s}, 2^{k}}(2 t) .
$$

When $t=1$, from Remark $6.2,2^{-(2 s-k)} c_{2^{s}, 2^{k}}(2)=1$, and the previous equality always holds.

When $t=2,3$, we know that, taking $\Sigma=X_{k-s}$, we do obtain a $2 t$-design, and hence, that (6.2) is fulfilled. We have proved two things:

- $2^{-(2 s-k) t} c_{2^{s}, 2^{k}}(2 t)=d_{k-s, k}(t-1)$.

- Assertion (2) of the theorem. 
It remains, of course, to give examples of sets $\Sigma$ with the property (2) of Theorem 6.3. One example is given by maximal spreads. These are standard objects of finite geometries.

Definition. The set $\Sigma \subset X_{w}$ is called a spread if $\Sigma$ is a set a totally isotropic subspaces, such that the intersection of two distinct elements is reduced to $\{0\}$. A maximal spread is a spread, such that $\cup_{S \in \Sigma} S$ is exactly equal to the whole set of isotropic elements.

The number of non zero isotropic vectors is $\left(2^{k}-1\right)\left(2^{k-1}+1\right)$. Therefore, a maximal spread in $X_{w}$ must have $\left(2^{k}-1\right)\left(2^{k-1}+1\right) /\left(2^{w}-1\right)$ elements, and hence a necessary condition for the existence of a maximal spread, is that this number is an integer. It is well known that, when $w$ divides $k$, maximal spreads do exist.

Theorem 6.4. Let $\Sigma$ be a maximal spread in $X_{k-s}$. Then, $\mathcal{D}_{\Sigma}$ is a 4design.

Proof. Let $\Sigma$ be a spread, and let $N:=|\Sigma|$. We calculate

$$
\frac{1}{|\Sigma|^{2}} \sum_{S, S^{\prime} \in \Sigma}\left|S \cap S^{\prime}\right|=\frac{1}{N^{2}}\left(N(N-1)+N .2^{k-s}\right)=1+\frac{2^{k-s}-1}{N} .
$$

On the other hand, from Remark 6.2,

$$
2^{-2(2 s-k)} c_{2^{s}, 2^{k}}(4)=\frac{2^{-(2 s-k)+1}}{3}\left(\frac{\left(2^{s}-1\right)^{2}}{2^{k}-1}+\frac{\left(2^{s-1}+1\right)^{2}}{2^{k-1}+1}\right) .
$$

The condition $(6.2)$ leads to $N=\left(2^{k}-1\right)\left(2^{k-1}+1\right) /\left(2^{k-s}-1\right)$.

Aknowledgements: I am indebted to Eiichi Bannai, Jacques Martinet, Gabriele Nebe, Neil Sloane for helpful discussions and comments.

\section{References}

[1] C. BaChoc, E. BannaI, R. Coulangeon, Codes and designs in Grassmannian spaces. Discrete Mathematics 277 (2004), 15-28.

[2] C. BACHOC, Linear programming bounds for codes in Grassmannian spaces. In preparation.

[3] C. Bachoc, R. Coulangeon, G. NeBe, Designs in Grassmannian spaces and lattices. J. Algebraic Combinatorics 16 (2002), 5-19.

[4] C. BACHOC, G. NEBE, Siegel modular forms, Grassmannian designs, and unimodular lattices. Proceedings of the 19th Algebraic Combinatorics Symposium, Kumamoto (2002).

[5] C. BACHOC, B. VenKov, Modular forms, lattices and spherical designs. In "Réseaux euclidiens, designs sphériques et formes modulaires", J. Martinet, éd., L'Enseignement Mathématique, Monographie no 37, Genève (2001), 87-111.

[6] E. BannaI, T. Ito, Agebraic Combinatorics I, Association Schemes (1984).

[7] M. Broué, M. Enguehard, Une famille infinie de formes quadratiques entières et leurs groupes d'automorphismes. Ann. Scient. E.N.S., $4^{e}$ série, 6 (1973), 17-52. 
[8] B. BolT, The Clifford collineation, transform and similarity groups III: generators and involutions. J. Australian Math. Soc, 2 (1961), 334-344.

[9] B. Bolt, T.G. Room, G.E. WALL, On Clifford collineation, transform and similarity groups I. J. Australian Math. Soc, 2 (1961), 60-79

[10] B. Bolt, T.G. Room, G.E. WALL, On Clifford collineation, transform and similarity groups II. J. Australian Math. Soc, 2 (1961), 80-96

[11] J. H. Conway, R. H. Hardin, E. Rains, P.W. Shor, N. J. A. Sloane, A group-theoretical framework for the construction of packings in Grassmannian spaces. J. Algebraic Comb. 9 (1999), 129-140.

[12] J. H. Conway, R. H. Hardin, N. J. A. Sloane, Packing Lines, Planes, etc., Packings in Grassmannian Spaces. Experimental Mathematics 5 (1996), 139-159.

[13] R. Coulangeon, Réseaux k-extrêmes. Proc. London Math. Soc. (3) 73 (1996), no. 3, 555-574.

[14] P. Delsarte, J. M. Goethals, J. J. Seidel, Spherical codes and designs. Geom. Dedicata 6 (1977), 363-388.

[15] P. Delsarte, V.I. Levenshtein, Association schemes and coding theory. IEEE Trans. Inf. Th. 44 (6) (1998), 2477-2504.

[16] R. Goodman, N. R. WALlaCh, Representations and invariants of the classical groups. Encyclopedia of Mathematics and its Applications 68, Cambridge University Press, 1998.

[17] A.T. James, A.G. Constantine, Generalized Jacobi polynomials as spherical functions of the Grassmann manifold. Proc. London Math. Soc. (3) 29 (1974), 174-192.

[18] J. Martinet, Sur certains designs sphériques liés à des réseaux entiers. In "Réseaux euclidiens, designs sphériques et formes modulaires, J. Martinet, éd., L'Enseignement Mathématique, Monographie no 37, Genève (2001).

[19] G. Nebe, W. Plesken, Finite rational matrix groups. Memoirs of the AMS, vol. 116, nb. 556 (1995).

[20] G. Nebe, E. Rains, N.J.A Sloane, The invariants of the Clifford groups. Designs, Codes, and Cryptography 24 (1) (2001), 99-122.

[21] G. Nebe, N.J.A Sloane, A catalogue of lattices. http://www.research.att.com/njas/lattices/index.html

[22] G. NeBE, B. Venkov, The strongly perfect lattices of dimension 10. J. Théorie de Nombres de Bordeaux 12 (2000), 503-518.

[23] G. NeBE, B. VenKov, The strongly perfect lattices of dimension 12. In preparation.

[24] B. Runge, On Siegel modular forms I. J. Reine Angew. Math. 436 (1993), 57-85.

[25] B. Runge, On Siegel modular forms II. Nagoya Math. J. 138 (1995), 179-197.

[26] B. Runge, Codes and Siegel modular forms. Discrete Math. 148 (1995), 175-205.

[27] D. Stanton, Some q-Krawtchouk polynomials on Chevalley groups. Amer. J. Math. 102 (4) (1980), 625-662.

[28] D. Stanton, Orthogonal polynomials and Chevalley groups. In Special functions: Group theoretical aspects and applications R.A. Askey, T.H. Koornwinder, W. Schempp editors, Mathematics and its applications, D. Reidel Publishing Company, 1984.

[29] B. Venkov, Réseaux et designs sphériques. In "Réseaux euclidiens, designs sphériques et formes modulaires, J. Martinet, éd., L'Enseignement Mathématique, Monographie no 37, Genève (2001).

[30] H. WEI, Y. WANG, Suborbits of the transitive set of subspaces of type $(m, 0)$ under finite classical groups. Algebra Colloq. 3:1 (1996), 73-84.

Christine BACHOC

Université Bordeaux I

351, cours de la Libération

33405 Talence, France

E-mail : bachoc@math.u-bordeaux1.fr

$U R L:$ http://www.math.u-bordeaux.fr/ bachoc 